\title{
The Psychological Experience of Obstetric Patients and Health Care Workers after Implementation of Universal SARS-CoV-2 Testing
}

\author{
Whitney R. Bender, MD ${ }^{1}$ Sindhu Srinivas, MD, MSCE ${ }^{1}$ Paulina Coutifaris, BA ${ }^{2}$ Alexandra Acker, BA $^{2}$
} Adi Hirshberg, $\mathrm{MD}^{1}$

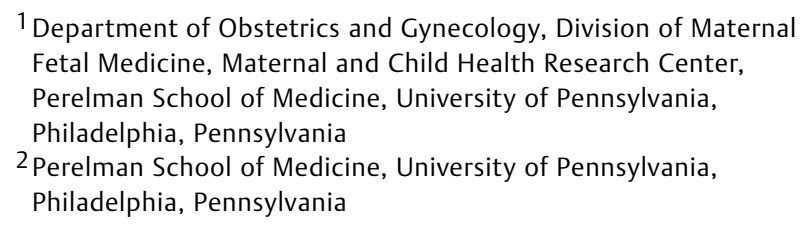

Address for correspondence Whitney R. Bender, MD, 3400 Spruce Street, Silverstein 2, Philadelphia, PA 19104

(e-mail: whitney.bender@pennmedicine.upenn.edu).

Am J Perinatol 2020;37:1271-1279.

\begin{abstract}
Objective This study was aimed to describe the hospitalization and early postpartum psychological experience for asymptomatic obstetric patients tested for severe acute respiratory syndrome-coronavirus-2 (SARS-CoV-2) as part of a universal testing program and report the impact of this program on labor and delivery health care workers' job satisfaction and workplace anxiety.

Study Design This is a cohort study of asymptomatic pregnant women who underwent SARS-CoV-2 testing between April 13, 2020 and April 26, 2020. Semistructured interviews were conducted via telephone at 1 and 2 weeks posthospitalization to assess maternal mental health. Depression screening was conducted using the patient health questionnaire-2 (PHQ-2). An online survey of labor and delivery health care workers assessed job satisfaction and job-related anxiety before and during the novel coronavirus disease 2019 (COVID-19) pandemic, as well as employees' subjective experience with universal testing. Patient and employee responses were analyzed for recurring themes.

Results A total of 318 asymptomatic women underwent SARS-CoV-2 testing during this 2week period. Six of the eight women (75\%) who tested positive reported negative in-hospital experiences secondary to perceived lack of provider and partner support and neonatal separation after birth. Among the 310 women who tested negative, $34.4 \%$ of multiparous women reported increased postpartum anxiety compared with their prior deliveries due to concerns about infectious exposure in the hospital and lack of social support. Only $27.6 \%$ of

Keywords

- COVID-19

- universal testing

- psychological experience

- postpartum experience

- mental health women, tested negative, found their test result to be reassuring. Job satisfaction and job-related anxiety among health care workers were negatively affected. Universal testing was viewed favorably by the majority of health care workers despite concerns about delays or alterations in patient care and maternal and neonatal separation.

Conclusion Universal testing for SARS-CoV-2 in obstetric units has mixed effects on maternal mental health but is viewed favorably by labor and delivery employees. Ongoing evaluation of new testing protocols is paramount to balance staff and patient safety with quality and equality of care.
\end{abstract}

received

June 21,2020

accepted after revision

July 13, 2020

published online

July 5, 2020
Copyright $\odot 2020$ by Thieme Medical Publishers, Inc., 333 Seventh Avenue, New York, NY 10001, USA. Tel: +1(212) 760-0888.
DOI https://doi.org/

10.1055/s-0040-1715505. ISSN 0735-1631. 
Key Points

- Women with SARS-CoV-2 had a negative hospital experience.

- A negative SARS-CoV-2 test was not reassuring for patients.

- COVID-19 negatively impacts healthcare workers' well-being.

The severe acute respiratory syndrome-coronavirus-2 (SARS-CoV-2) pandemic has dramatically changed our world. Social isolation, job insecurity and unemployment, increased childcare and homeschooling responsibilities, and fear of illness have contributed to a growing sense of anxiety and discomfort, as well as mental health crises, throughout the United States.

Labor, delivery, and the early postpartum period are a time of significant change and stress; concerns about postpartum blues and depression have existed long before the pandemic. The need for continued interactions with the health care system to obtain ongoing pregnancy-related care during the pandemic places pregnant patients in a uniquely vulnerable position. In addition to the concern for viral acquisition through these interactions, pregnant patients' vulnerability to adverse mental health outcomes may be exacerbated by the lack of usual in-person family and social support during pregnancy, delivery, and the postpartum period in the current pandemic. This phenomenon has yet to be quantified or described in the medical literature. Specifically, while the benefits of universal SARS-CoV-2 testing on labor and delivery units to optimize staff and patient safety have been published, the impact of a universal SARS-CoV-2 testing program on maternal stress or anxiety has not been reported. ${ }^{1-3}$

In addition to the toll of SARS-CoV-2 on obstetric patients' psychological experiences, health care workers exposed to SARS-CoV-2 are also at increased risk for symptoms of depression, anxiety, insomnia, and distress. ${ }^{4}$ Data from past outbreaks suggest that clear communication, access to adequate personal protective equipment (PPE), and adequate rest and support may mitigate these ill effects. $^{5}$ The impact of universal SARS-CoV-2 testing on the mental health of health care workers on labor and delivery units, however, is unclear.

The objectives of this study were to (1) describe the hospitalization and early postpartum psychological experience for asymptomatic obstetric patients tested for SARSCoV-2 as part of a universal testing program and (2) report the impact of universal SARS-CoV-2 testing on labor and delivery health care workers' job satisfaction and workplace anxiety.

\section{Materials and Methods}

This is a cohort study of pregnant women presenting for obstetric care at two hospitals within a large academic health system in Philadelphia, PA, between April 13, 2020 and April 26, 2020. This time frame was chosen as it was the first 2 weeks of universal SARS-CoV-2 testing at these hospitals. Health care workers on these units were also included in this study. This study was deemed exempt by the University of Pennsylvania Institutional Review Board.

All women presenting for delivery at the two hospitals were verbally consented for testing for SARS-CoV-2 via nasopharyngeal and oropharyngeal swab as part of a universal testing clinical protocol. A real-time polymerase chain reaction (PCR) assay was performed for the qualitative detection of nucleic acid of the SARS-CoV-2 virus. Women who declined testing or were unable to be tested for medical reasons were considered uninfected. Women who declined testing were excluded from this study. Women admitted for other obstetric indications and deemed likely to deliver during the admission were also tested. Women who reported viral symptoms such as cough, fevers/chills, or anosmia, were febrile at the time of presentation, or had a known SARS-CoV-2 exposure were deemed persons under investigation by the admitting obstetrical provider and were excluded from this study, specifically evaluating asymptomatic patients with no known exposure. Patients with prior positive SARS-CoV-2 test results were also excluded.

While on labor and delivery, patients who tested positive for SARS-CoV-2 received obstetric care with use of appropriate PPE by all employees. They were allowed one support person during labor, but this person was not allowed to remain with them during postpartum recovery. Women who tested positive were transferred to a private room on a separate SARS-CoV-2-dedicated unit with ongoing care from both obstetric and internal medicine teams. After delivery, women and neonates were separated for the duration of the hospitalization as per institutional protocol. Neonates remained in the newborn nursery or neonatal intensive care nursery as appropriate. Pumped breastmilk was utilized for infant feeding for breastfeeding mothers. Neonates were tested at 24 hours of life and again prior to discharge. At discharge, women were not instructed to social distance from their neonates. They were asked to wear surgical masks with feeding or close infant contact and instructed on frequent hand washing.

Women who tested negative for SARS-CoV-2 were allowed to have one support person for the entirety of their delivery and postpartum recovery. They remained in the usual postpartum unit. Private rooms were utilized preferentially, and semiprivate rooms were used as needed for census. Patients and their support persons were asked to wear masks during all interactions with hospital staff. Health care workers and hospital employees wore face masks at all times during the study time period.

Test results, demographic, obstetric, and neonatal data were abstracted from the electronic medical record (EMR) by a single investigator (W.B.). Data were stored in RedCap. 
Semistructured interviews assessing for symptom development in the patients or family members of patients were conducted via telephone at 7 to 9 and 12 to 14 days posthospital admission and test date as part of routine clinical follow-up for the universal testing program. Telephone calls were performed by a maternal-fetal medicine fellow and two medical students. These individuals received written instructions on interview conduct. Instructions included use of the standardized script described below and written verbatim transcription of subject responses. Recording devices were not used for any telephone calls. The patient health questionnaire-2 (PHQ-2) was also verbally administered during each phone call; women with positive screens were referred for mental health services. Non-English speaking women were contacted using a telephone medical interpretation service.

Interview questions varied according to parity and SARSCoV-2 test result. Nulliparous women were asked "How has fear or anxiety regarding novel coronavirus disease 2019 (COVID-19) impacted your ability to bond with or care for your baby and recover from delivery?" Multiparous women were asked, "Compared to your prior deliveries, how has fear or anxiety of COVID-19 altered your postpartum experience?" Women who tested negative for SARS-CoV-2 were asked "In the hospital you tested negative for COVID-19. How did this impact any fear or anxiety you had during your hospitalization?" Women who tested positive for SARS-CoV2 were asked, "In the hospital, you tested positive for COVID19. How did this change your labor, delivery, and postpartum experience?" All women were asked "Is there anything that could have been done prior to your hospital admission to improve your hospital experience?"

Questions were modified for women who did not deliver on the index hospitalization as neonatal care and maternal recovery were not applicable. These undelivered women were asked "How has COVID-19 impacted your feelings about hospitalization for delivery?" These women were asked the same questions about SARS-CoV-2 testing and hospital experience improvement as described above.

Patient responses to the open-ended questions that were documented in the medical record at time of telephone call. Three authors (A.A., P.C., and W.B.) reviewed the telephone calls as documented in the medical record and recorded subject responses in a separate document. This document was analyzed by all three authors for common themes. Meaningful direct patient statements were also extracted.

As part of the universal testing implementation process on labor and delivery, health care workers providing care on the two hospital units completed an online survey and were asked to rate their job satisfaction and job-related anxiety prior to and since the SARS-CoV-2 pandemic. They were asked to reflect upon the impact of the universal testing program on these metrics and were also given the opportunity to share their experience working during the pandemic with open-ended questions. These responses were transcribed directly as written by the respondent and analyzed for common themes and meaningful statements in a similar fashion to patient responses.
Sample size was fixed based on the number of women admitted during the prespecified timeframe. Descriptive statistics were calculated for the population at large. The association of categorical variables with binary outcomes were analyzed using Fisher's exact test or Chi-squared analyses as appropriate. The associations of continuous variables with binary outcomes were analyzed using student's $t$-test or Wilcoxon's rank-sum tests for nonnormally distributed variables.

\section{Results}

A total of 318 women were tested for SARS-CoV-2 during the specified 2-week timeframe. Seven women did not undergo SARS-CoV-2 testing and were excluded from further analysis and follow-up. Of the 318 women, 301 women delivered a liveborn neonate. Ten women were discharged without delivery, and seven women delivered a stillborn neonate. These women are included in the final cohort. Black women accounted for $43 \%$ of the total population. Sixty percent of the population used private insurance and $47 \%$ were nulliparous.

\section{Asymptomatic Women with a Positive SARS-CoV-2 Test}

Eight of the 318 women (2.5\%) were positive for SARS-CoV-2. Six delivered a liveborn neonate. One was discharged undelivered, and one delivered a stillborn neonate. Five of the eight women were nulliparous. No women required escalation of clinical care, and all were discharged home within usual obstetric discharge timing, based on mode of delivery. All eight women were reached for follow-up at 1 week posthospitalization; seven were reached for 2-week follow-up.

When asked about their experience after testing positive for SARs-CoV-2, three women, including the woman who delivered a stillborn, reported no concerns regarding their hospitalization. Among the five women who reported negative feelings about their hospitalization, there were two common themes summarized in -Table 1. The first theme was a feeling of neglect or isolation from staff and support people. The second centered on neonatal separation after birth. Despite these experiences, only two women reported feeling additional fear or anxiety in the postpartum period secondary to their diagnosis. Only one woman reported any recommendations for improving the hospital experience and simply suggested "more support."

In addition, all women who tested positive for SARS-CoV2 were asked the PHQ-2 at the time of each phone call. At 1week post-hospitalization, one woman (12.5\%) reported feeling down, depressed, or hopeless at least half the days of the last 2 weeks, and no one reported feeling little interest or pleasure in doing things at this time. At the 2 -week phone call, all women surveyed had PHQ-2 scores of $\leq 1$.

\section{Asymptomatic Women with a Negative SARS-CoV-2 Test}

A total of 310 women tested negative for SARS-CoV-2. One hundred and sixty-six women (53.5\%) were multiparous. 
Table 1 Themes identified in asymptomatic SARS-CoV-2 test positive women

\begin{tabular}{ll}
\hline Theme & Quotations \\
Neglect, isolation & "Felt I needed more attention" \\
& "Felt like no one wanted to come around me. It was hard to be by myself with no support" \\
& "Felt neglected by the staff. The lactation consultant called over phone rather than coming in the \\
& "Wom to try to explain how to pump" \\
"Worse time of my life..., while trying to experience something that was supposed to be beautiful" \\
neonatal separation
\end{tabular}$\quad \begin{aligned} & \text { "I never got any updates on my baby unless I asked" } \\
& \text { "It was so hard to be apart from my son while in the hospital" } \\
& \text { "Feel like I didn't get to enjoy the birth because of separation. I feel like I didn't 'do right' by him }\end{aligned}$

Abbreviation: SARS-CoV-2, severe acute respiratory syndrome-coronavirus-2.

Two hundred and forty-two (78.1\%) of these women were reached for a follow-up phone call at 1 week posthospitalization, and 213 women (68.7\%) were reached for a follow-up phone call at 2-week posthospitalization. A complete list of the identified themes from these phone calls can be found in -Table 2.

In the multiparous group, when asked if COVID-19 led to additional fear or anxiety in the postpartum period when compared with previous deliveries, 34.4\% responded "yes." Among these patients, a common source of their anxiety was fear of becoming infected with COVID-19 during their hospitalization due to contact with potentially sick patients or providers. Another source of anxiety was the inability to have friends and family visit and help care for their infant. One patient stated, "we don't want anyone around us at home. Whereas with my other baby, everyone was here all the time." When nulliparous women were asked if fear or anxiety regarding COVID-19 impacted their ability to bond with or care for their baby or recover from delivery, $100 \%$ replied "no."

When patients who tested negative for COVID-19 were asked if their negative test result changed any fear or anxiety they had during their hospitalization, $27.6 \%$ reported relief or reassurance, whereas $72.4 \%$ said testing negative did not change their level of fear or anxiety. Within this cohort, many stated that their anxiety did not change because they continued to be hospitalized for several days after learning of their test result and thus remained at risk for infection. Others stated that the negative test result did not change their anxiety because they were confident that they did not have COVID-19 due to precautions that they took preceding delivery. Interestingly, one patient reported that, while it didn't change her level of fear or anxiety, she hoped "it would change the way health care providers would interact with (me)."

When asked if there was anything that could have been done prior to admission to the hospital that would have improved their hospital and postpartum experience, 8.9\% suggested improvements whereas $91.1 \%$ were satisfied. Common suggestions included dissemination of hospital COVID-19 policies, such as lack of partner testing and visitor rules in advance of admission, as well as enhanced communication between patients and providers. Some also stated that during their hospitalization they were concerned about close contact with other patients, availability of private rooms, and shared waiting areas. Among those women who were satisfied with their experience, there was an attitude of appreciation for the safety measures implemented, such as temperature checking and symptom screening, as well as general respect for the health care team "doing their best."

At the 14-day follow up, asymptomatic patients with negative SARS-CoV-2 test results were asked "Since we last spoke, what changes have there been in your fear or anxiety regarding COVID-19 and being postpartum?" Between the 7and 14-day follow-up, $96.2 \%$ of patients reported no change, $2.8 \%$ reported feeling less anxious, and $0.98 \%$ reported feeling more anxious. A common theme among those who felt less anxious was that a sufficient amount of time had passed since their discharge from the hospital without experiencing COVID-19 symptoms. For example, one patient said, "I feel more relieved that I'm past the 14-day mark and we are well and good."

In addition, patients were asked the PHQ-2 at the time of each phone call. At one week posthospitalization, 5 women (2\%) of the 242 contacted reported little interest in doing things at least half of the days in the last 2 weeks, while 8 women (3.3\%) reported feeling depressed at least half the days in that same time period. Repeat administration of the PHQ-2 also occurred at 2-week posthospitalization. At this time, 5 women $(2.3 \%)$ of the 213 reached reported a little interest in doing things at least half of the days in the last 2 weeks, while 8 women (3.8\%) reported feeling depressed at least half the days in that same time period.

\section{Labor and Delivery Employees}

One hundred and fifty-eight (37.4\%) labor and delivery employees of the 422 recipients responded to the survey. Sixty-six registered nurses responded, comprising $42 \%$ of those surveyed. Fifty-four (34\%) of survey respondents were attending physicians, advanced practice providers, or midwives, and 29 (18\%) were resident physicians. Six (4\%) obstetrical anesthesia attendings also participated in the survey. The remainder of the respondents were divided amongst operating room technicians and unit secretaries.

-Fig. 1 shows job satisfaction data as reported before and during the SARs-CoV-2 pandemic. Prior to the pandemic, 93.0\% (147) employees reported feeling very satisfied or 
Table 2 Themes identified in asymptomatic SARS-CoV-2 test negative women

\section{Theme}

Fear of infection in the hospital; relief of this fear when again at home and isolating.

Fear of leaving the house postpartum/causing infant infection

Difficulty with social isolation from family and friends with a new baby

Relief at testing negative for SARS-CoV-2

Confidence in their ability to test negative for SARS-CoV-2

Fear about testing positive for SARS-CoV-2

Desire for additional testing

Improvement in fear/anxiety as additional time from hospitalization passes

\section{Quotations}

"Obviously you're afraid you're going to get it"

"It was scary to be in a hot spot where I know people are going to get care for the virus"

"We're more concerned about whether we came into contact with anything in the hospital"

"It was obviously more nerve racking being in a hospital environment"

"I was actually scared to go to the hospital because I thought that was where I had the highest chance of being exposed"

"My recovery in the hospital was poor because of anxiety regarding COVID-19"

"I was very anxious about getting infected during my hospital stay due to contact with sick patients or providers. I feel much better now that I'm home and able to isolate"

"The nurses did a good job but at the end of the day you have anxiety when being around other people in the hospital. It's just praying that we're not developing symptoms and going to come out fine"

"My throat is sore from being intubated and, even though they told me that would happen, I'm still worried I have COVID-19 every time I feel it"

"I'm more on edge about taking him to the pediatrician"

"I'm fearful of going to the store and bringing something back to my baby"

"And you're afraid you're going to get the baby sick. It's awful"

"I can't take the baby outside and have anxiety about getting him to his next appointment" "There's concern... being exposed to people... like going to the grocery store and not bringing it back home to baby"

“I'm scared because my babies are really small and they're immunocompromised. I'm nervous all the time. I literally have anxiety now. I just want them to be ok. I'm nervous when it comes to newborns anyway but now it's like triple thousands"

“I'm just trying to make sure everyone stays safe. It's nerve racking, making sure he's as safe as possible. It's just a lot"

"It's very challenging to not have visitors especially because we have another baby in the NICU" "It has prevented us from having help from outside. It's been pretty hard but we're getting by" "It's terrifying to let anyone come near your child"

"It's scary because everyone is so excited about the kids but I can't let family over to the house and I'm nervous"

"I had some pretty had baby blues and I don't have the support system that I had before"

"It's tough. It's been really hard. I'm so used to my support system so not having them around is really hard"

"It changed my level of anxiety a lot"

"It was reassuring that I could not pass infection to the baby"

"Knowing that the hospital was testing everyone, as well as my negative test, made me more relaxed"

"Once I took the test, I was put at ease that me and baby would be ok"

"Knowing I was negative let me relax and stop worrying about passing something on to my baby"

"I expected to be negative because I had been social distancing for several weeks"

"I knew I was negative because I had no symptoms and was self-quarantining"

"I didn't have symptoms so I didn't think I was sick"

"I had known I was home and wasn't concerned about being positive"

"I knew I didn't have COVID-19."

"I was being really careful and quarantined for almost 2 months aside from doctors' visits... I would have been shocked (to test positive)"

"I was afraid of testing positive and the repercussions of that"

"I had anxiety going into the hospital and knowing I was going to be tested. Before having the result it was anxiety provoking"

"I mean I was more anxious waiting for the results... ."

"(It was) strange that my partner didn't get tested"

"I didn't know the status of the medical personnel or my husband. I didn't think I was positive anyways but to not know the status of other people can cause anxiety"

"I wish they would have tested us again 14 days after we left the hospital so we know if we were exposed"

"Social distancing and being home away from the healthcare setting has been good"

"I would say it's a little better with every day we get further away from being in the hospital"

"The more days go by the better I feel because I don't feel ill"

Abbreviations: COVID-19, novel coronavirus disease 2019; NICU, neonatal intensive care unit; SARS-CoV-2, severe acute respiratory syndromecoronavirus-2. 


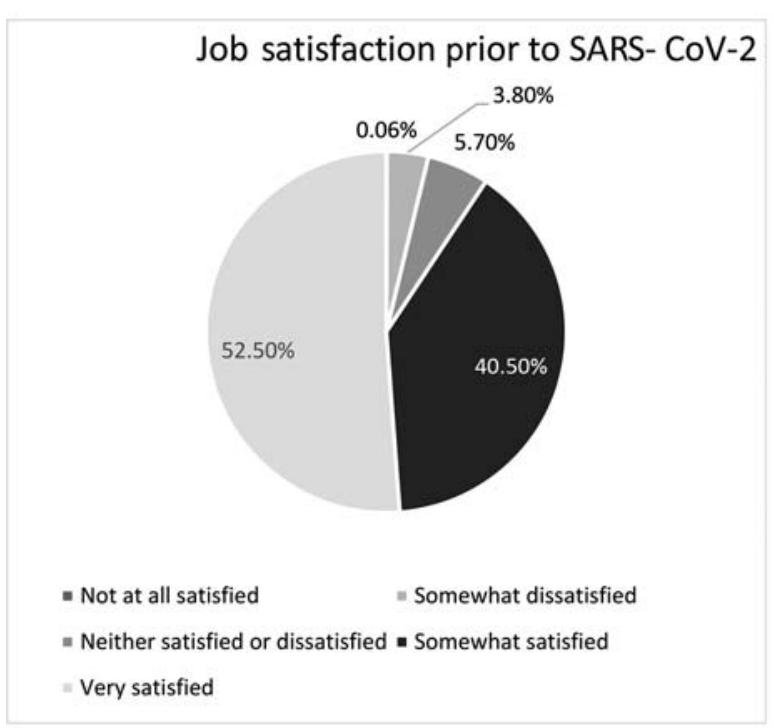

Job satisfaction since SARS-CoV-2
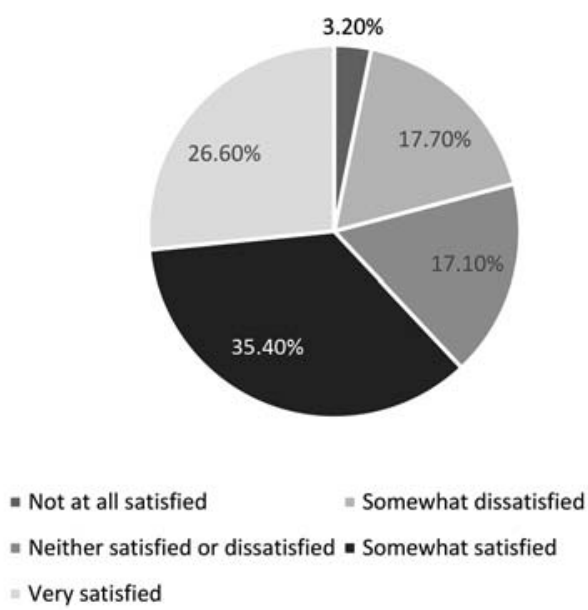

Fig. 1 Job satisfaction before and after SARS-CoV-2 among labor and delivery employees. SARS-CoV-2, severe acute respiratory syndromecoronavirus- 2 .

somewhat satisfied with their job. This figure dropped to $62.0 \%$ after the pandemic started. Significantly more employees reported being less than "somewhat satisfied" after the pandemic started ( 10.1 vs. $40.0 \%, p<0.0001)$. In addition to these changes in job satisfaction, there were corresponding changes to job-related anxiety as seen in - Fig. 2 . Prior to the pandemic, the majority of respondents $(n=100,62 \%)$ reported minimal job anxiety. In contrast, $54 \%(n=85)$ reported moderate job anxiety since the pandemic. Additionally, the proportion of respondents reporting significant job anxiety increased substantially from 1 to $27 \%$. $(p<0.0001)$. When asked about the impact of universal SARs-CoV-2 testing on these metrics, $61 \%(n=96)$ of respondents reported an increase in job satisfaction and 66\% $(n=104)$ reported a decrease in job-related anxiety.

As part of this survey, employees were also asked to comment on the impact of universal SARs-CoV-2 testing on timeliness and quality of care. A complete list of themes and direct quotations about their experiences with universal testing can be found in -Table 3. Sixty-three (39.9\%) respondents reported witnessing delays in care secondary to universal testing, such as delays in the start of scheduled inductions or cesarean sections, as well as increased time to anesthesia evaluation in women in whom tests were still pending. Forty-six percent $(n=77)$ of respondents reported changes in care provided secondary to universal SARs-CoV-2 testing. In addition to expected changes in isolation and personal protective equipment for those testing positive, respondents reported concerns regarding mode of obstetric care and neonatal care. One respondent wrote "(delivering) your baby to providers in full PPE feels different." Another stated "I have seen providers apply different induction of labor methods to patients with COVID-19." Respondents also commented on the impact of universal testing on patient/provider communication, with concerns that providers were spending less time at the bedside.
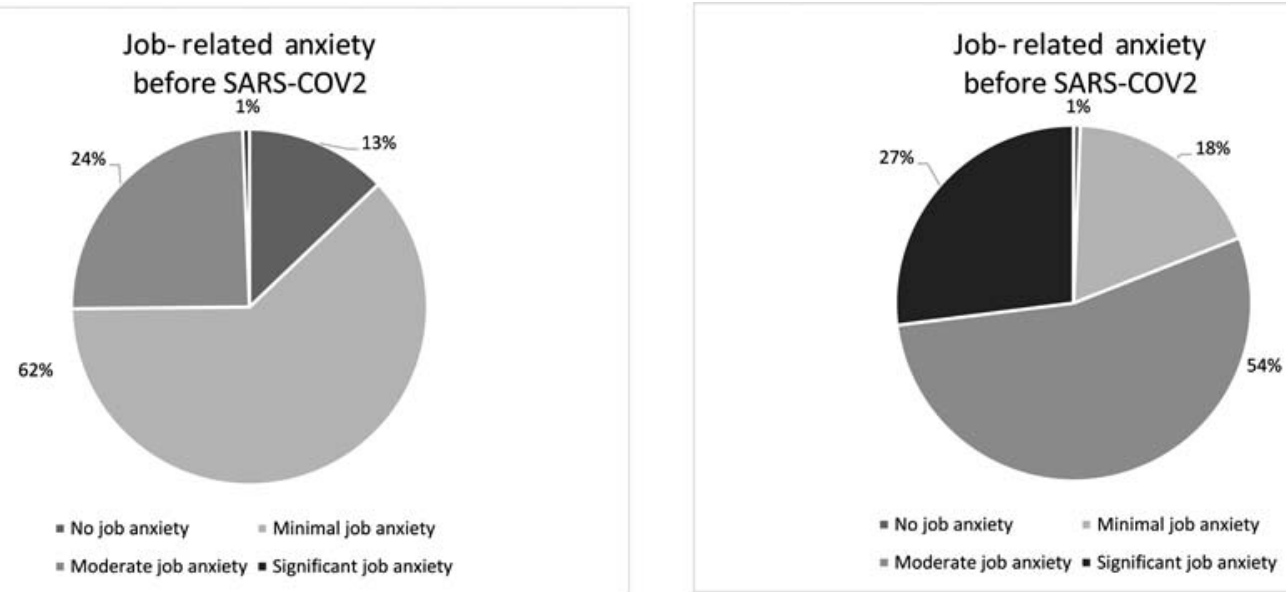

Fig. 2 Job-related anxiety before and after SARS-CoV-2 among labor and delivery employees. SARS-CoV-2, severe acute respiratory syndromecoronavirus-2. 
Table 3 Themes identified in labor and delivery employees

\begin{tabular}{|c|c|}
\hline Theme & Quotations \\
\hline $\begin{array}{l}\text { Changes in care related to } \\
\text { universal SARS-CoV-2 } \\
\text { testing or SARS-CoV-2 } \\
\text { status }\end{array}$ & $\begin{array}{l}\text { "Impacts the methods of induction as well as the number of interactions with providers" } \\
\text { "I don't think they get as thorough MD care" } \\
\text { "If not willing to be screened, providers are less willing to spend time at patients' bedside" } \\
\text { "Less time spent in COVID-19+ rooms" } \\
\text { "Decreased contact within room. Patient expressed feeling separated or a "black sheep"” } \\
\text { "Positive patients have less frequent checks and likely less provider interactions" } \\
\text { "Not deniable that having to deliver your baby to providers in full PPE feels different" } \\
\text { "Often providers are desiring to do telephone encounters instead of an in-person HPI." }\end{array}$ \\
\hline $\begin{array}{l}\text { Delays in care related to } \\
\text { universal SARS-CoV-2 } \\
\text { testing or SARS-CoV-2 } \\
\text { status }\end{array}$ & $\begin{array}{l}\text { "In one instance, a COVID positive patient with a category II tracing had providers slow to make } \\
\text { decisions due to her COVID status. I felt like if it were any other patient, she would have been a } \\
\text { (STAT) C-section." }\end{array}$ \\
\hline $\begin{array}{l}\text { Concerns regarding neo- } \\
\text { natal separation }\end{array}$ & $\begin{array}{l}\text { "I am bothered by the pediatric policy of separating mom from baby in asymptomatic patients. } \\
\text { It seems fairly arbitrary given that many of these patients go home on PPD } 1 \text { with baby" Missing } \\
\text { those first } 24 \text { hours is so key to establishing breastfeeding and many other things" } \\
\text { "Frustrated by universal infant separation from asymptomatic positive mothers, when they } \\
\text { will be discharged home with their babies in } 24-48 \text { hours unprepared to care for their babies } \\
\text { with minimal guidance" } \\
\text { "I'm not sure if I would agree to testing given the risk of being separated from my baby" }\end{array}$ \\
\hline $\begin{array}{l}\text { Negative Impact if } \\
\text { patients decline testing or } \\
\text { test positive }\end{array}$ & $\begin{array}{l}\text { "Patients who are positive or refuse testing create anxiety among staff caring for them" } \\
\text { "Bias and fear/uncertainty in those who decline testing" } \\
\text { "When people have refused testing, they are labeled and likely cared for in a different maybe } \\
\text { more biased manner" } \\
\text { "If they refuse the swab, there is a stigma from L\&D staff and I think we can improve in terms of } \\
\text { our attitude" } \\
\text { "They are declining something that can make me feel safer" }\end{array}$ \\
\hline $\begin{array}{l}\text { Desire to provide better } \\
\text { care if SARS-CoV-2 test is } \\
\text { negative }\end{array}$ & $\begin{array}{l}\text { "I am less anxious and feel more comfortable helping them in their labor" } \\
\text { "I feel that it brings both the patient and provider a sense of comfort during a crazy time... I } \\
\text { believe a negative test result allows me to be a little more hands on" }\end{array}$ \\
\hline $\begin{array}{l}\text { Positive impact of univer- } \\
\text { sal SARS-CoV-2 testing on } \\
\text { health care worker }\end{array}$ & $\begin{array}{l}\text { "The universal COVID-19 testing gives me a greater peace of mind, knowing that I am } \\
\text { protecting myself" } \\
\text { "Having every patient admitted to labor and delivery be tested for COVID-19 has eased anxiety } \\
\text { and made me feel safer at work" } \\
\text { "Universal testing really helped with the anxiety of being close to the patient" } \\
\text { "The universal testing makes me feel safer doing my job" } \\
\text { "It increased safety for health care workers, not just anxiety and satisfaction" }\end{array}$ \\
\hline $\begin{array}{l}\text { Desire for additional } \\
\text { testing }\end{array}$ & $\begin{array}{l}\text { "It has been helpful to have universal testing of the patient, but it hasn't reduced my anxiety } \\
\text { much because the partners are not screened" }\end{array}$ \\
\hline
\end{tabular}

Abbreviations: COVID-19, novel coronavirus disease 2019; HPI, history of present illness; L\&D, labor and delivery; MD, maternal delivery; PPD1, postpartum day 1; PPE, personal protective equipment; SARS-CoV-2, severe acute respiratory syndrome-coronavirus-2.

Respondents also reflected on the impact of testing on their own anxiety or patient anxiety. Negative results were viewed favorably among respondents as "providing reassurance" or allowing for "usual treatment." A "stigma from labor and delivery staff" was suggested toward women who declined testing, although this was an infrequent occurrence during the 2-week study time frame. One respondent reported that these women "are labeled and likely cared for in a different maybe more biased manner."

Of the 66 respondents who left additional comments, 36 (54.5\%) expressed their appreciation for universal testing and desire for its continuation.

\section{Discussion}

In this retrospective study of universal SARS-CoV-2 testing in obstetric patients, the majority of women who tested positive for SARS-CoV-2 reported a negative hospital experience secondary to perceived lack of care and institutional recommendation for neonatal separation. Despite these experiences, however, only $25 \%$ of positive women reported increased fear or anxiety in the postpartum period and no women who tested positive for SARS-CoV-2 had a positive depression screen on the PHQ-2 at 2-week postpartum.

Among women who tested negative, there was a mixture of gratitude for a negative test and anxiety about potential exposures in the hospital after this negative test. The anxiety related to SARS-CoV-2 decreased as time from hospital discharge increased. Although suggested by multiple patients, it is unclear how repeated negative testing at hospital discharge would have affected this trajectory. The inability to have family member support due to the pandemic contributed to more anxiety in the multiparous patients. 
This heightened sense of anxiety and isolation was widely reported, and yet very few women screened positive on the PHQ-2. This suggests that additional questions may need to be asked of newly postpartum women to fully understand the complexity of their feelings in this pandemic.

Labor and delivery employees reported a negative impact on job satisfaction and job-related anxiety secondary to the SARS-CoV-2 pandemic, consistent with reports from COVID19 positive units in China. ${ }^{4}$ Universal testing on labor and delivery was self-reported to have improved these metrics. Despite reporting delays in care, changes in obstetric care, and patient/provider communication, survey respondents reported very positive feelings on universal testing likely secondary to decreased employee anxiety. Despite the perception of altered care, no adverse maternal or neonatal outcomes occurred during this period attributable to SARS-CoV-2 test status. Continued evaluation of intended and unintended consequences on care, however, is important as more units implement universal testing.

As described by women who tested positive for SARS$\mathrm{CoV}-2$, the separation of mother and baby in cases of COVID-19 caused significant distress. These feelings of anxiety and grief were echoed by labor and delivery survey respondents. The recommendation for maternal-neonatal separation is based on current concern regarding close contact resulting in neonatal viral acquisition and illness. A shared decision-making approach incorporating patient desires should be considered as hospital policy makers balance the importance of maternal and neonatal bonding in the immediate postpartum period with neonatal health and safety. Our institution is considering modifying our approach based on the results of this survey and patient feedback.

As we continue to care for SARS-CoV-2 positive and negative women during the pandemic, we must exercise caution in balancing patient and provider safety with quality and equality of care provided. Providers should be aware of the possibility for biased treatment based on test results or acceptance of testing and do their best to recognize and rectify its presence. Careful incorporation of telemedicine conversations may be utilized to minimize provider exposure to SARS-CoV-2 positive patients. However, clinical judgment should be used in determining what clinical information can be appropriately communicated in this manner and what information warrants an in-person evaluation. Additionally, all hospital staff should be aware of ongoing practice changes secondary to SARS-CoV-2 and given the opportunity to provide their feedback on their implementation.

\section{Limitations and Strengths}

This study has several strengths. This was a large cohort study of all asymptomatic pregnant women presenting for care at two hospitals in an academic health system. It is the first to systematically collect and describe the emotional impact of the SARS-CoV-2 pandemic and universal testing on pregnant patients. The inclusion of the health care workers' perspective is an important addition when describing the consequences of universal SARS-CoV-2 testing. The reported improvement in job satisfaction and job-related anxiety supports institution-wide patient testing and consideration of partner testing, if able, as has occurred in other delivery units. $^{6}$

This study is not without limitations. As this study was conducted within a single academic health system, it may not be generalizable to the population at large. Additionally, this data are from women during the first 2 weeks of the universal testing initiative and, as such, may not be representative of the experiences of women receiving care later in the pandemic. Seven women did not undergo testing during the study period. These women were excluded from this follow-up study, and, as such, their perspectives are not included in the telephone surveys. Given the retrospective nature of data collection, we were unable to further characterize any of the patient-reported experiences. Additionally, as telephone calls were recorded via text only, we are unable to account for any paraphrasing or omissions of participant answers. Although subjective responses did not seem to vary by parity, race, insurance, or mode of delivery, the number of women in these subsets were too small to make any conclusions about individuals whose experience may be more affected by factors other than their SARS-CoV-2 test result. Additionally, our test-positive rate is lower than reported for other geographic regions. It is unclear how the patient and health care worker experience would differ had the infection rate been higher.

Despite these limitations, there are important lessons to be taken from this study. The majority of women testing positive for SARS-CoV-2 had a negative hospital experience specifically related to their care experience and infant separation. This underscores the importance of ongoing evaluation of how new care processes impact patients, as we do our best to balance staff and patient safety with quality of care and experience. The impact of negative testing on patients was mixed. Even with negative testing, many postpartum women continue to experience anxiety at home. Specific questions on this topic may be necessary to elicit these emotions and to continue to develop innovative yet safe ways to provide our patients with the support and care needed during these difficult times. Lastly, universal SARS$\mathrm{CoV}-2$ testing at the time of delivery hospitalization improves employee satisfaction and anxiety. These lessons can be applied broadly as institutions incorporate universal testing strategies to prioritize the health of their patients and the wellbeing of their staff.

Conflict of Interest

None declared.

\section{References}

1 Sutton D, Fuchs K, D'Alton M, Goffman D. Universal screening for SARS-CoV-2 in women admitted for delivery. N Engl J Med 2020; 382(22):2163-2164

2 Breslin N, Baptiste C, Gyamfi-Bannerman C, et al. Coronavirus disease 2019 infection among asymptomatic and symptomatic 
pregnant women: two weeks of confirmed presentation to an affiliated pair of New York City hospitals. Am J Obstet Gynecol MFM 2020;2(02):100118

3 Campbell KH, Tornatore JM, Lawrence KE, et al. Prevalence of SARS-CoV-2 among patients admitted for childbirth in Southern Connecticut. JAMA 2020;323(24):2520-2522

4 Lai J, Ma S, Wang Y, et al. Factors associated with mental health outcomes among health care workers exposed to coronavirus disease 2019. JAMA Netw Open 2020;3(03):e203976
5 Kisely S, Warren N, McMahon L, Dalais C, Henry I, Siskind D. Occurrence, prevention, and management of the psychological effects of emerging virus outbreaks on healthcare workers: rapid review and meta-analysis. BMJ 2020;369:m1642

6 Buckley A, Bianco A, Stone J. Universal testing of patients and their support persons for COVID-19 when presenting for admission to labor and delivery within the Mount Sinai Health System. Am J Obstet Gynecol MFM. 2020 (e-pub ahead of print). Doi: 10.1016/j. ajogmf.2020.100147 